\title{
Traducción revisada por la autora: la semiautotraducción en La casa de los conejos de Laura Alcoba ${ }^{1}$
}

\author{
María Eugenia Ghirimoldi \\ mghirimoldi@fahce.unlp.edu.ar \\ Universidad Nacional de La Plata
}

\section{Resumen}

Dentro de la diversidad de situaciones donde el autor interviene en las traducciones, el presente trabajo propone indagar entre las fronteras de la autotraducción, la retraducción y la semiautotraducción, para describir el espacio que habita nuestro caso de estudio. Analizamos las transformaciones de semiautotraducción identificadas en La casa de los conejos, la traducción al español de la novela Manèges. Petite histoire argentine, en su edición aniversario revisada por la autora, Laura Alcoba, a diez años de la publicación de la primera traducción realizada por Leopoldo Brizuela. En un primer momento, recorremos las diversas definiciones de autotraducción, retraducción y semiautotraducción. Esta última noción es la que adoptamos para caracterizar la obra traducida estudiada. En una segunda etapa, examinamos las transformaciones detectadas en la nueva traducción en comparación con las opciones de la primera. Para ello, adaptamos las categorías propuestas por Patricia López LópezGay, con el fin de crear una tipología propia para el presente análisis. De los datos recogidos pueden observarse tres tendencias: la primera es la de aspirar a una mayor precisión con respecto al original en comparación con la primera traducción; la segunda tendencia consiste en introducir elementos nuevos que no aparecen en el texto original, y la tercera, la de introducir algunos elementos típicos de la variedad de español rioplatense argentino. Este estudio invita a reflexionar sobre las traducciones con participación del autor, así como poner a prueba las categorías de análisis existentes.

Palabras clave: autotraducción; La casa de los conejos (Laura Alcoba); retraducción; semiautotraducción; traducción literaria; transformaciones de traducción.

\section{Traduction revue par l'auteure : la semiautotraduction} dans La casa de los conejos de Laura Alcoba

\section{Résumé}

Parmi la diversité des situations où l'auteur intervient dans les traductions, le présent travail propose d'enquêter sur les frontières de l'autotraduction, la retraduction et la semiautotraduction afin de décrire l'espace qui habite notre cas d'étude. Nous avons analysé les transformations de semiautotraduction identifiéés dans La casa de los conejos (2018), la traduction à l'espagnol du roman Manèges. Petite histoire argentine (2007), dans l'édition anniversaire révisée par l'auteure, Laura Alcoba,

1 Este artículo surge del proyecto "Multimodalidad, géneros discursivos y circulación de las ciencias humanas y sociales: un abordaje crítico desde la traductología y la didáctica de las lenguas extranjeras", Universidad Nacional de La Plata, 2020-2024, Código H944. 
dix ans après la publication de la première traduction réalisée para Leopoldo Brizuela, La casa de los conejos (2008). En premier lieu, nous parcourons les différentes définitions d'autotraduction, de retraduction et de semiautotraduction. C'est cette dernière notion celle que nous adoptons pour caractériser l'œuvre traduite étudiée. Ensuite, nous examinons les transformations repérées dans la nouvelle traduction, produit de la comparaison avec les choix de la première traduction. Pour cela nous avons adapté les catégories proposées par Patricia López López-Gay (2008) afin de créer notre propre typologie pour la présente étude. D'après les données recueillies il est possible d'en relever trois tendances. La première vise une plus grande précision par rapport à l'original si on compare avec la première traduction. La deuxième tendance consiste à introduire de nouveaux éléments qui ne se lisent pas dans le texte d'origine. La troisième tendance perçue c'est celle d'introduire quelques éléments typiques de la variété d'espagnol rioplatense en Argentine. Cette étude invite à réfléchir sur les traductions revues par l'auteur ainsi qu'à mettre à l'épreuve les catégories d'analyse existantes.

Mots clés : retraduction ; autotraduction ; semiautotraduction ; transformations de traduction ; $L a$ casa de los conejos.

\title{
Author-Revised Translations: The Semi-Self-translation of La casa de los conejos, by Laura Alcoba
}

\begin{abstract}
Within the diversity of situations where the author collaborates in translations, the present work proposes to investigate between the frontiers of self-translation, retranslation and semi-self-translation to describe the space that our case study inhabits. It looks into the transformations identified in the semi-self-translation La casa de los conejos (2018), the translation into Spanish of the novel Manèges. Petite histoire argentine (2007). Ten years after the publication of the first translation by Leopoldo Brizuela, La casa de los conejos (2008), this translation emerges as the anniversary edition revised by the author, Laura Alcoba. This paper reviews the different definitions of self-translation, retranslation, and semi-self-translation, this latter category adopted for the analysis of the translation. Further, the transformations identified in this new translation are examined vis-à-vis the first translation. For the purposes of the analysis, a typology is created based on the categories proposed by Patricia López López-Gay (2008). Three trends have been identified from the data collected. Firstly, this translation pursues more precision relative to the source text than the first translation. Secondly, changes are introduced to this new version as compared to the source text. Finally, there is a tendency to introduce some typical elements of rioplatense Spanish. This study invites reflection on translations in collaboration with the authors, as well as on the need of problematizing existing categories of analysis.
\end{abstract}

Keywords: retranslation; self-translation; semi-self-translation; translation transformations; La casa de los conejos. 


\section{Introducción}

El presente trabajo de investigación en traducción literaria se ubica dentro de territorios cada vez más explorados en traductología, en especial desde fines del siglo $\mathrm{xx}$ hasta la actualidad: los de la retraducción y la autotraducción. Los casos de estudio de traducciones revisadas por el autor, al compartir características de la retraducción y la autotraducción, aportan elementos para repensar estas categorías que, en la práctica, se desplazan creando formas combinadas.

Defendemos la idea de que en este tipo de traducciones donde interviene el autor, la libertad que le permite su posición se desplaza entre dos polos, entre alejamiento y acercamiento al texto original, es decir, entre la recreación libre, que introduce cambios con respecto al original, y los ajustes de imprecisiones detectadas en la primera traducción, en vistas a ganar un mayor acercamiento al original.

Para poner a prueba nuestras hipótesis, en el apartado 2 recorremos las nociones teóricas acerca de las diversas categorías de la mano de referencias indiscutibles como Antoine Berman (1990), Xosé Manuel Dasilva (2016), Rainier Grutman (1998, 2009), Julio César Santoyo (2005), Helena Tanqueiro (1999, 2007), entre otros, donde establecemos las relaciones con el caso estudiado.

Luego, en el apartado 3, abordamos la metodología para analizar y comparar las traducciones, inspirada en las técnicas de traducción de Amparo Hurtado Albir (2013) y Lucía Molina (2006); en las nociones de "error de traducción" de Jeanne Dancette (1989), Jean Delisle (1993), Juan Carlos Palazuelos et al. (1992), y en las llamadas "transformaciones de traducción", de Patricia López López-Gay (2008).

En la sección 4 analizamos las transformaciones de semiautotraducción identificadas en $L a$ casa de los conejos (2018a), publicada por la editorial argentina Edhasa, en su edición aniversario revisada por la autora, a diez años de la publicación, en la misma editorial, de la primera traducción al español realizada por el escritor Leopoldo Brizuela (2008) de la novela Manèges. Petite histoire argentine de Laura Alcoba (2007), publicada por la editorial francesa Gallimard.

En la última sección, presentamos nuestras conclusiones a partir de los análisis comparativos de las traducciones entre sí y en relación con el original, y comentamos las tendencias identificadas que caracterizan la traducción revisada por la autora.

Este estudio sobre una revisión de traducción efectuada por la reconocida autora y traductora franco-argentina contribuye a poner a prueba las categorías de análisis, a ejemplificar las matizadas tendencias en las modificaciones llevadas a cabo y a adoptar el término de "semiautotraducción" (Dasilva) para describir el caso particular que nos ocupa.

La novela elegida para este trabajo es el primer eslabón de una trilogía de corte autobiográfico, de la niñez a la adolescencia, junto a Le bleu des abeilles (2013) o El azul de las abejas (2015), traducción de Leopoldo Brizuela, y a La danse de l'araignée (2017) o La danza de la araña (2018b), traducción de Mirta Rosenberg y Gastón Navarro.

La casa de los conejos narra treinta años después, a través de la ficcionalización de la voz de la niña Laura, las vivencias de la infancia en la clandestinidad. Entre los años 1975 y 1976, la niña vive junto a su madre y una pareja de militantes montoneros, en una casa donde funcionó la imprenta clandestina del periódico Evita montonera, oculta tras la fachada de un criadero de conejos. Luego de haberse exiliado en Francia, Alcoba tuvo noticias del brutal bombardeo de los militares a la casa y el trágico destino de las personas que la habitaban, entre ellas Diana Teruggi, a quien dedica esta obra.

La beba de Diana, Clara Anahí, se supone fue apropiada por los militares, y se ha convertido en símbolo de los desaparecidos que pudieron 
haber quedado con vida, pero secuestrados bajo otra identidad. María Isabel Chicha Chorobik de Mariani (1923-2018), la abuela de Clara, fue una de las fundadoras de la Asociación Abuelas de Plaza de Mayo, cuyo objetivo es encontrar a los niños desaparecidos en la última dictadura.

\section{Recorrido teórico sobre la autotraducción, la retraducción y la semiautotraducción}

El caso que elegimos para analizar, la versión en español de 2018 de Manèges, presenta varios desafíos en términos de categorización, por tratarse de una traducción donde la información sobre el trabajo de revisión de la autora no se muestra en la portada.

En esta edición estudiada sigue figurando, en la portada interior, el nombre del traductor Leopoldo Brizuela, como en la primera edición en español de 2008, y la intervención de la autora en calidad de revisora de la traducción solo se visibiliza de manera explícita en la contratapa. Estamos frente a una nueva edición de la obra traducida al español, fruto de la labor de un traductor y de una autora que interviene en el proceso de la traducción.

Para poder caracterizar este caso particular de traducción con colaboración de la autora, recorreremos los diversos conceptos de autotraducción, retraducción y semiautotraducción.

\subsection{Autotraducción}

La autotraducción, definida por Anton Popovic en 1976 como la traducción que realiza el propio autor de una obra original a otra lengua, es un fenómeno intercultural cuyo estudio ha sido reivindicado desde fines del siglo xx por autores como Grutman $(1998,2009)$ — quien escribió la entrada "Auto-translation" para la enciclopedia de traductología de Routledge en 1998-, Francesc Parcerisas i Vazquez (2007), López López-Gay (2008), Tanqueiro $(1999,2007)$, entre muchos otros.
Santoyo (2005) habla a menudo de "traducciones de autor". Por su parte, Parcerisas las describe como un "proceso por el cual un autor dado vierte su obra en otra lengua" (2007; nuestra traducción).

Se destaca, en este tipo de reescritura que elaboran los autores sobre sus propias obras, el poder que tienen para recrear o revisar sus versiones libremente, y a los cuales se les considera, siguiendo la categoría de Tanqueiro, "traductores privilegiados" (1999), beneficio del que no goza el traductor de una obra ajena. Es innegable que no hay "nadie como el propio autor, nadie con más autoridad que él para resolver las dudas y responder a las preguntas que todo traductor se hace cuando se enfrenta a las sutilezas de un texto ajeno" (Santoyo, 2005, p. 864).

En relación con este privilegio de los autotraductores, López López-Gay subraya que, en la autotraducción, el autor goza de un margen de libertad, destinado principalmente a adaptar su obra al nuevo sistema cultural, y que, a su vez, dicha libertad está vinculada a su pertenencia a un ámbito editorial y académico, donde autores y traductores no ostentan los mismos poderes (López López-Gay, n. d., p. 4). Este aspecto, relacionado con nuestra hipótesis de partida, será discutido más adelante luego de nuestro análisis.

La autotraducción se vincula con frecuencia con autores que escriben en el exilio, como el caso de la autora elegida para este estudio. Como señala Grutman, referencia ineludible en el tema, este desplazamiento espacial, sea impuesto o voluntario, da origen a la actividad autotraductiva. Debido a este pasaje de fronteras territoriales y lingüísticas, Grutman propone denominarlos escritores "translingües" (Grutman, 2009, p. 117), apelando a la partícula latina trans (del otro lado, más allá), en tanto se trata de quienes han dejado atrás un territorio y una lengua, y han debido adaptarse a la tierra de acogida (Grutman, 2013, p. 40). Estos escritores que escriben en otras lenguas distintas de 
su lengua materna se encuentran, en términos de Georges Steiner, en "extraterritorialidad" (2009, p. 6). En este caso, su bilingüismo es exógeno, extranjero a su tierra natal.

Para Grutman, existen, por un lado, los autotraductores exógenos, los que se tradujeron luego de su desplazamiento y, por otro, los endógenos, aquellos provenientes de sociedades diglósicas (2007). Teniendo en cuenta esta categorización de autotraductores sugerida por Grutman a partir de su análisis de los escritores que se autotradujeron (Santoyo, 2002, p. 30; 2005 , p. 864), proponemos ubicar a nuestra autora analizada dentro de estos parámetros. Alcoba escribe como escritora exógena, porque se desplaza a París desde Argentina, exiliada a los 10 años, para reencontrarse con su madre; pero al recibir casi toda su educación en el país y en la lengua de acogida, escribe la totalidad de sus obras en la lengua adoptada. De esto resulta que, adaptando estas categorías al caso estudiado aquí, su (auto)traducción o, mejor dicho, la revisión de la traducción alógrafa, en su propia lengua materna, la inscribe en la subcategoría de (auto)traductora exógena que escribe fuera de su tierra, que elige escribir en la lengua otra y se (auto)traduce a su lengua primera.

El caso de Alcoba, como el de muchos otros escritores translingües (Alexakis, Beckett, Joyce, Kundera, Nabokov), desafía la idea romántica según la cual la cuestión de cómo un autor habría escrito sus obras en otra lengua no tendría que ser siquiera formulada, dado que nadie escribe una obra original sino en su primera lengua (Schleiermacher, 2000).

Ahora bien, Alcoba no escribe en español; todas sus obras fueron redactadas en francés. Al igual que numerosos autores provenientes sea del exilio, o de minorías culturales, como lo expresa María Laura Spoturno, "traducen sus obras literarias recurriendo a originales culturales, es decir, discursos asociados a la lengua que indica el origen cultural, que no existen sensu stricto" (2010, p. 1246). La escritura de Alcoba en lengua francesa es, sin duda, producto de la traducción de su propia identidad cultural, traducción de sus memorias ficcionalizadas vividas en su país de origen en su lengua primera, el español. Esta idea está ligada al concepto de autotraducción mental o in mente propuesto por Tanqueiro (2011), un proceso de traducción simultáneo al de la escritura de la propia obra original, donde los autores tratan universos socioculturales diferentes a los de los lectores del original.

Para Alcoba, como para muchos escritores bilingües, "la lengua es una zona de conflicto, deseo y creatividad" (Spoturno, 2018, p. 359; nuestra traducción). Laura nació en 1968 en Cuba y llegó al mes y medio con sus padres a la ciudad de La Plata, provincia de Buenos Aires, Argentina, donde fue registrado su nacimiento. El español es su lengua primera, pero la lengua callada, la lengua en la que no podía expresarse con libertad, la lengua del silencio de sus años de infancia en la década de los setenta, vivida bajo la clandestinidad, por ser hija de militantes montoneros, en la época de la última dictadura militar en Argentina, que se extendió desde 1976 hasta 1983. E1 francés es la lengua aprendida desde los 7 años como preparación para su futuro exilio y en su nueva vida en Francia, a partir de 1979, lengua que le permite tomar la distancia emocional necesaria para poder contar aquel pasado traumático.

\subsection{Retraducción}

Luego de haber establecido relaciones de la autotraducción con el caso estudiado de revisión de la autora, pasamos a indagar sobre las que podemos detectar con la actividad de retraducción. Según Yves Gambier, "la retraducción sería una nueva traducción, en una misma lengua, de un texto ya taducido, completo o en parte" (1994, p. 413, nuestra traducción). Antoine Berman propuso un concepto más amplio, según el cual "basta con que un texto de un autor haya sido traducido para que 
la traducción de los otros textos de este autor entre en el espacio de la retraducción" (1990, p. 4, nuestra traducción).

La retraducción suscita muchos interrogantes, debido a su carácter polimorfo, consecuencia de las múltiples funciones que en ella cumple el traductor. Este término lo desarrolló Liliane Rodriguez (1990), cuando comparó las principales funciones atribuidas al dios Mercurio con las del traductor, como las de mensajero, servidor, inventor de las letras, gimnasta, vendedor, ladrón y viajero. Cada retraducción tiene origen, de acuerdo con Rodriguez, en alguna de estas funciones mercurianas de la retraducción, que emerge, según el caso, de manera combinada y en un proceso dinámico con las demás funciones (1990, pp. 63-80).

Trasladando esta perspectiva a nuestro análisis, la tarea de retraducción llevada a cabo por Alcoba combina de manera predominante dos funciones: por un lado, la función del vendedor, en tanto procura que nada se pierda, en especial salvaguardar los pequeños detalles del original que se pudiesen haber desviado en la primera traducción, donde, además, la nueva edición en la cual se publica la retraducción pasa a constituirse en un argumento de venta; por otro, la función del mensajero, que siente la necesidad de adaptar el mensaje a otro momento (diez años más tarde) y a otros lectores (argentinos).

El caso que nos ocupa también tiene su origen en la sensación de insatisfacción, por parte de la autora, con respecto a la primera traducción. Las íntimas razones que llevan a un escritor a retraducir no pueden ser reveladas en su totalidad. En general, se van construyendo a partir de los dichos del autor en sus entrevistas y de lo que puede inferirse de su discurso literario.

En cuanto a las motivaciones reveladas explícitamente por la autora que la impulsaron a revisar la primera traducción, comentaremos sus dichos, registrados personalmente en ocasión de una conferencia en Angoulême, Francia, en 2018. Allí explica que su lengua de escritura es el francés y por ese motivo reconoce la necesidad de acudir a un traductor para trasladar su obra al español. Para la escritora, es extraordinario que el traductor y escritor argentino Brizuela le haya propuesto la tarea de traducir Manèges. Pero, a su vez, Alcoba admite la dificultad de aceptar completamente la versión traducida y considera necesario opinar y proponer cambios, de acuerdo con sus declaraciones durante una entrevista personal inédita (Alcoba, comunicación personal telefónica, 15 de marzo de 2021).

Este deseo de transformación, de intervenir en aquella traducción primera, tuvo su origen cuando recibió la propuesta de hacer una lectura pública para un espectáculo con música de Jean-Jacques Lemêtre y Ariane Mnouchkine, en Argentina. Junto con la directora de teatro Clara Bauer, Laura realizó un découpage y cuando comenzaron los ensayos, percibieron que el tono no era el adecuado, que no se escuchaba la voz infantil como en la versión francesa, y entonces se hicieron los primeros cambios.

Según explicó durante el mencionado intercambio, la autora sintió la necesidad de hacer pequeñas modificaciones, al percibir ciertos problemas de registro y algunas expresiones. Pero destacó que no es una nueva traducción, sino una traducción revisada, retocada, que llevó a cabo en solitario a partir del trabajo de Brizuela, que este aceptó y autorizó. En efecto, él es el traductor que firmó la publicación revisada, puesto que Alcoba no quiso que pusieran su nombre indicando esta tarea de revisión.

Luego de esa experiencia, Alcoba propone al editor de Edhasa poder hacer transformaciones en la totalidad del libro, y es allí donde comienza el proyecto de la edición aniversario, a diez años de la primera publicación, donde se publicaría la nueva versión revisada por la autora. 
La nueva traducción revisada muestra, además, otra característica que acompaña generalmente a las retraducciones: la intertextualidad. Los paratextos señalan su estatus y hacen explícita la interpretación que el retraductor inscribe en su nueva traducción. Estos pueden consistir en introducciones y epílogos, anotaciones, comentarios, el respaldo de académicos especialistas, entre otros. En el caso estudiado aquí, la inclusión de un prólogo de la pluma de Daniel Pennac, que se anuncia en la tapa, es una estrategia literaria y editorial. Titulado "El libro de los silencios", este paratexto se encarga de afirmar una ideología, la que reivindica el símbolo que representa en Argentina la actuación de madres, padres, abuelas y abuelos que reclaman a los hijos e hijas asesinados o desaparecidos durante la última dictadura. También se destaca la vida en clandestinidad de la niña Laura, se elogia la precisión de la escritura en francés de la autora, y en el último párrafo se anuncia sutilmente que se trata de una nueva traducción con colaboración de la escritora: "Volver a llevar La Casa de los conejos al castellano en ocasión del décimo aniversario de su publicación, es celebrar de la manera más bella el reencuentro de Laura con Alcoba" (p. 12; énfasis en el original). Como se señaló antes, es en la contratapa del libro donde se indica, de manera explícita, que se trata de una traducción revisada por la autora.

\subsection{Semiautotraducción}

Entre las diferentes nociones de traducción con participación del autor, consideramos la de semiautotraducción, desarrollada por Xosé Manuel Dasilva (Dasilva, 2016, p. 24), la que mejor se ajusta para caracterizar el presente caso. En su recorrido por diversas definiciones de la autotraducción donde el autor no es el único responsable, de la autotraducción con colaboración alógrafa a la semiautotraducción, Dasilva recoge las propuestas de diversos autores, ilustrando la dificultad de hallar una denominación única para una actividad altamente heterogénea.
Por su parte, María Recuenco Peñalver diferencia entre "(auto)traducción autorial" y "(auto) traducción parcialmente autorial". Dentro de esta última distingue, a su vez, entre "(auto) traducción parcialmente autorial revisora" y "(auto)traducción parcialmente autorial revisada" (Recuenco Peñalver, 2013, p. 84). E1 primer caso, el de (auto)traducción parcialmente autorial se acercaría más al nuestro de Laura Alcoba, donde la autora parte de una traducción alógrafa, la realizada por el traductor Brizuela en 2008, y la somete a cambios.

Como explica Dasilva en su artículo de 2016, Josep Miquel Ramis Llaneras diferencia entre "autotraducción directa" y "autotraducción indirecta", siendo la segunda la opción en que el autor ayuda a un traductor alógrafo, y otros, como Elizabete Manterola Agirrezabalaga (2014, p. 93), señalan varios niveles en el tipo de colaboración, donde ambos trabajan mano a mano o donde el traductor lleva a cabo la traducción y luego el autor revisa el escrito.

E1 término "semiautotraducción" que propone Dasilva (2016, p. 26) para dar cuenta de la compleja heterogeneidad de los textos traducidos donde el autor interviene en alguna medida, aunque no de modo completo, resulta apropiado para caracterizar el presente caso de estudio. Entre las modalidades que describe de semiautotraducción, el nuestro, el de la segunda versión, se corresponde al de traducción alógrafa revisada por el autor. En cuanto al grado de participación en el proceso de traducción entre las dos partes, no se trata aquí de una labor mano a mano, sino de un trabajo en solitario de la autora que, a partir de la primera traducción, revisó el escrito.

\section{Metodología para el análisis de las transformaciones de la semiautotraducción}

Para analizar las transformaciones observadas resultantes de la comparación del original en francés con las dos traducciones al español en el caso en estudio, indagamos acerca de diversas taxonomías aplicables a los fenómenos 
encontrados. En primer lugar, para conocer las diferencias entre los textos, los criterios que seguimos se basaron en la clasificación de las técnicas de traducción propuestas por Amparo Hurtado Albir (2013) y Lucía Molina (2006); además, recorrimos las nociones de "error de traducción" (Dancette, 1989; Delisle, 1993; Palazuelos et al., 1992), que permiten tipificar los fenómenos de interpretación errónea de un segmento del texto origen en una traducción (falso sentido, adición, omisión, hipertraducción, sentido diferente, sentido restringido). A estas nociones, para describir las modificaciones encontradas, les sumamos, por último, las llamadas "transformaciones de traducción" de López López-Gay (2008).

Inspirándose en las transformaciones de traducción de Popovic (2011), que permiten apreciar las modificaciones del texto traducido con respecto al original, es decir, "aquello que es nuevo en relación con el original, o que no se encuentra donde 'debería' encontrarse" (López López-Gay, n. d., p. 8), la mencionada investigadora propone la noción de "formas de transformación de la autotraducción", para aquellas realizadas por la traducción, que remitena la doble posición que el autor-traductor ocupa en el campo literario. Estas formas básicas son tres: 1) adiciones: toda forma de ampliación; 2) supresiones: toda forma de omisión, y 3) sustituciones: combinación supresión-adición (López López-Gay, 2008, p. 188).

En su artículo sobre la autotraducción como reflexión, López López-Gay (n. d., p. 4) identifica tres planos en que se manifiesta la intervención del autotraductor: el plano lingüístico formal, el plano de la reelaboración (corrección de errores tipográficos, históricos, incluso de contenido textual) y el plano cultural ideológico (adaptación al nuevo receptor, cuyo sistema cultural el autor conoce bien).

López López-Gay ilustra la tendencia, en la autotraducción, a presentar un grado mayor de precisión y de desarrollo de ideas (n. d., p. 8). En relación con la precisión, se observa un extremo cuidado en los detalles, mayor exactitud, claridad, explicitación. Con respecto al grado de desarrollo de ideas y argumentaciones, se añade información suplementaria, adiciones de tipo cultural, aunque también puede aparecer el efecto contrario, que alguna información del original desaparezca en la traducción.

Basándonos en la nomenclatura de López López-Gay, luego de observar nuestro corpus, el original en francés y las dos traducciones al español, hemos reunido las transformaciones observadas en lo que llamaremos aquí "transformaciones de la semiautotraducción" en tres categorías. Por un lado, adoptamos dos de las tendencias propuestas por López López-Gay: en primer lugar, la de alcanzar una mayor precisión que en la primera traducción con respecto al original; en segundo lugar y menos marcada, la de introducir elementos nuevos en la segunda versión, no existentes en el original. Por otro lado, agregamos una tercera tendencia, la de introducir elementos léxicos propios de la variedad de español rioplatense hablada en Argentina. En la Tabla 1 mostramos la taxonomía de subcategorías, de cosecha propia, dentro de cada una de las tres tendencias.

El procedimiento de análisis consistió, en primer lugar, en identificar, luego de comparar la primera traducción y la traducción revisada, las transformaciones que revelaban las diferencias entre los textos. Se revisaron los textos completos frase por frase. La categorización de las transformaciones se presenta en comparación con la primera traducción. En segundo término, cotejamos de manera simultánea el original con las dos versiones.

El corpus textual fue construido y analizado con una planilla de cálculo. Dentro de cada categoría, propusimos subcategorías y mostramos los fragmentos encontrados, ofreciendo el ejemplo en el original en francés, luego la primera traducción al español y, por último, la propuesta de la revisión, señalando en negrita las diferencias y el número de página al final del ejemplo entre paréntesis. Se indicaron, durante 
Tabla 1. Transformaciones de la semiautotraducción

\begin{tabular}{|c|c|c|}
\hline \multirow{3}{*}{$\begin{array}{l}\text { Mayor precisión con respecto al } \\
\text { texto original }\end{array}$} & 1. Mayor acercamiento al original & $\begin{array}{l}\text { Eliminación de agregados de la } \\
\text { primera traducción } \\
\text { Mayor neutralización } \\
\text { Registro menos vulgar } \\
\text { Restitución de términos con carga } \\
\text { ideológica }\end{array}$ \\
\hline & $\begin{array}{l}\text { 2. Reposición de elementos } \\
\text { eliminados en la primera traducción }\end{array}$ & \\
\hline & 3. Ajustes de sentido & $\begin{array}{l}\text { Sentido diferente } \\
\text { Sentido restringido } \\
\text { Falso sentido } \\
\text { Sentidos metafóricos }\end{array}$ \\
\hline \multirow{4}{*}{$\begin{array}{l}\text { Introducción de elementos nuevos } \\
\text { no presentes en el texto original }\end{array}$} & 1. Explicitación & \\
\hline & 2. Pérdida del cambio de lenguas & \\
\hline & 3. Omisiones & $\begin{array}{l}\text { Eliminación de referencias a las } \\
\text { variedades de lenguas española } \\
\text { Omisiones de expresiones con } \\
\text { carga ideológica }\end{array}$ \\
\hline & 4. Adiciones & \\
\hline \multirow{4}{*}{$\begin{array}{l}\text { Introducción de elementos léxicos } \\
\text { del español de Argentina }\end{array}$} & 1. Tiempos verbales & $\begin{array}{l}\text { Pretérito indefinido } \\
\text { Futuro próximo }\end{array}$ \\
\hline & 2. Introducción del "vos" & \\
\hline & 3. Omisión del pronombre sujeto & \\
\hline & $\begin{array}{l}\text { 4. Variedades de español americano } \\
\text { y argentino }\end{array}$ & $\begin{array}{l}\text { Afectivos } \\
\text { Coloquial } \\
\text { Regionales }\end{array}$ \\
\hline
\end{tabular}

el análisis, los textos del corpus y las fuentes consultadas.

\section{Análisis de las transformaciones de semiautotraducción}

En este apartado analizamos las transformaciones o categorías de lo que hemos dado en llamar "semiautotraducción" (en adelante, SAT) observadas en la segunda traducción de La casa de los conejos, revisada por la autora del original y publicada por Edhasa en 2018, y que resultan de la comparación con las opciones de la primera traducción alógrafa $(\operatorname{Tr} 1)$, publicada en 2008.

\subsection{Transformaciones de mayor precisión con respecto al texto original}

Los cambios realizados en la traducción revisada se caracterizan por alcanzar una mayor precisión con respecto a la obra original en francés, mediante diversos mecanismos que dividimos en: mayor acercamiento al texto original (Tо), reposición de elementos eliminados en la Tr1 y ajustes de sentido, que describimos a continuación.

\subsubsection{Mayor acercamiento al texto original}

Los cambios que se orientan a ganar mayor precisión con respecto al to se concretizan por medio de diversas estrategias: eliminación de agregados en la primera traducción, neutralización, registro menos vulgar y restitución de términos ideológicamente marcados.

1. Eliminación de los agregados de la primera traducción: "cette petite fille"(p. 74), que deviene "esa pobre nena" (p. 67) en la Tr1 —donde el traductor adicionó el adjetivo ausente en el original-, vuelve 
en la SAT al sentido más literal de la autora: "esa nena" (p. 69).

2. Neutralización: cambios de las opciones ideológicamente cargadas en la Tr1, por otras más neutras en la SAT. La frase "les hommes des AAA" (p. 76) en el TO es traducida en la Tr1 por "los 'monos' de las "Tres AAA""2 (p. 69), donde la elección de "monos" en lugar de "hombres" tiñe el concepto peyorativamente, y le agrega color local, debido a que este término se usa en Argentina para designar o bien a una persona de poco seso y afectado por sus modales (Zorrilla, 2018), o bien, en un registro popular, a los policías uniformados y guardaespaldas, entre otros usos. En la SAT se vuelve a la expresión más neutra, por modificar el término "monos" por "los hombres de la Triple A" (p. 72), ajustando, además, la redundancia de la expresión "Tres AAA".

3. Registro menos vulgar: cambio de los términos más vulgares de la Tr1 por otros más formales y cercanos al To. La expresión en el To: "Il faut arrêter, là!" (p. 111), es traducida como "¡Pero paremos, carajo!" (p. 101), donde se introduce una palabra vulgar, "carajo", que denota enfado, sorpresa o insulto, y en la SAT se vuelve al tono más formal con “¡Pero basta, ya!” (p. 103).

4. Restitución de términos con alto contenido ideológico, neutralizados en $\operatorname{Tr} 1$ : "un lieu de mémoire" del to (p. 137) pasa, en la $\operatorname{Tr} 1$, a "espacio de recordación" (p. 127). La SAT repone la expresión "espacio de memoria" (p. 129) con peso propio, pues evoca resonancias especiales en la historia. Los espacios de memoria son lugares públicos que hoy están destinados de manera permanente a la transmisión de

$2 \mathrm{La}$ Triple A alude a la Alianza Anticomunista Argentina, organización de extrema derecha que sembró el terror con sus asesinatos, entre 1973 y 1975. la memoria, la educación y la promoción y defensa de los derechos humanos.

Otro ejemplo. En el To dice: "elle a été enlevée par les militaires" (p. 139); en Tr1: "fue criada por militares" (p. 128), mientras en la SAT: "fue secuestrada por los militares" (p. 130). De este modo, la autora repone el sentido del то con su transformación en la SAT, puesto que "enlever" significa, en este contexto, "secuestrar, raptar a una persona". En español se puede asociar la carga del original en francés a la del verbo "chupar", usado en el contexto de las prácticas de desaparición forzada e ilegal de personas durante la dictadura. En Tr1 se cambió el sentido, suavizándolo y privándolo de la tremenda carga negativa que transmite el término en el original.

\subsubsection{Reposición de fragmentos eliminados en la Tr1}

El siguiente fragmento: "Cela s'est passé ainsi pour des centaines d'autres. Quelques-uns d'entre eux ont été retrouvés. D'autres sont encore recherchés par leur famille : Clara Anahí est de ceux-là" (p. 140), es omitido en la Tr1. En la SAT aparece: "Centenas de bebés conocieron el mismo destino. Muchos de ellos fueron encontrados. Los familiares siguen buscando a muchos otros, como a Clara Anahí" (p. 131). La SAT repone la traducción de esta información, desgarradora, que restaura un contexto real, donde la autora subraya, trayéndolos a la memoria, el reclamo y la denuncia por estos hechos.

Otro ejemplo de segmento omitido corresponde a un diálogo de la narradora con Chicha Mariani, la abuela de Clara Anahí, sobre el paradero del personaje del Ingeniero:

-Il est où, maintenant?

—Là-dessus, différentes hypothèses circulent également. Certains disent qu'il est en Australie, d'autres parlent de l'Afrique du Sud. Mais j'ai rencontré quelqu'un qui m'a dit qu'il avait été tué par les militaires eux-mêmes après tout $c ̧ a$ (p. 142). 
En la sAT se repone así:

- ¿Y dónde está ahora? -Al respecto, existen varias hipótesis. Algunos dicen que vive en Australia, otros hablan de África del Sur. También conocí a alguien que dice que los propios militares lo mataron después de todo esto (p. 133).

Este contenido, altamente significativo para la historia contada, expone las diversas hipótesis que alimentan el misterio sobre el destino del Ingeniero, el que creó el dispositivo de cierre electrónico de la puerta "invisible" de la imprenta clandestina y uno de quienes se sospechó que, quebrado bajo la tortura, delató la ubicación de la casa que fue bombardeada por los militares.

Un ejemplo más: en el To aparece: "Je veux croire aussi qu'il y a bien d'autres 'excessives évidences"' (p. 145), que es omitido en la Tr1, y se repone en la SAT, así: "Quiero creer que hay muchas otras 'excesivas evidencias"' (p. 136). Esta idea, que expresa la intención de dejar en suspenso la duda, que ofrece un matiz en las últimas reflexiones sobre la posible traición del Ingeniero, es restituida por la autora en la SAT.

Por último, "Il avait su déchiffrer les lettres énormes. Les gros caracteres" (p. 144) en el To, se vierte, en Tr1, de forma recortada: "Él supo descifrar las letras enormes" (p. 133). En la SAT, se repone dicha omisión: "Él pudo descifrar las letras enormes. Las mayúsculas" (p. 135).

\subsubsection{Ajustes de sentido}

En esta subcategoría de transformaciones de semiautotraducción que buscan alcanzar mayor precisión en relación con el original, observamos cuatro tipos de ajuste de sentido: corrección en la SAT de sentido diferente, de sentido restringido, de falso sentido y de sentido metafórico empleados en la primera traducción.

1. Sentido diferente: "volant" (p. 18), en el TO, hace referencia al volante de un ómnibus. En Tr1 se tradujo con un sentido diferente, el de "manubrio" (p. 15), que, según el Diccionario la lengua española, de la Real Academia Española (DRAE), refiere a una empuñadura o pieza que se emplea para dar vueltas a una rueda. En la SAT se reemplaza por "volante" (p. 17).

2. Sentido restringido: "une grande personne" (p. 20) en el To, en Tr1 se traduce con un sentido restringido, el de "una persona mayor" (p. 17), y en la SAT se cambia por "una adulta" (p. 20). Persona mayor es aquella de sesenta años o más; en cambio, una persona adulta se acerca más al sentido utilizado en el texto, el de una persona que ha alcanzado su desarrollo pleno.

3. Falso sentido: en la Tr1 se tradujo "quelques mois avant la guerre des Malouines" (p. 134) por "desde poco después de la Guerra de Malvinas" (p. 124), y en la SAT se repone el sentido del original, "unos meses antes de la guerra de Malvinas" (p. 126).

4. Sentido metafórico: la oración en el то: "Je me suis envolée pourl'Argentine" (p. 137) es traducida en Tr1: "Me fui volando a la Argentina" (p. 127); y en la SAT: "Tomé un avión a Argentina" (p. 129). El verbo en francés "s'envoler" significa "partir en avión" (Larousse). En la Tr1 se cambia el sentido literal por uno metafórico, el de "salir volando a algún lado". Según el DRAE, uno de los sentidos de "volar" es "caminar o ir con prisa y aceleración". En la sAT se repone el sentido del original, que es "tomar un avión".

Asimismo, se corrige el uso del artículo delante de los países, obligatorio en francés, que se elimina en español.

Encontramos otro ejemplo de este tipo de ajuste. En el To, dentro de "certains clients de mon grandpère savaient aussi renvoyer l'ascenseur comme des princes, à l'occasion" (p. 134), se utiliza una expresión con sentido figurado "renvoyer l'ascenseur", 
empleada cuando una persona recibe un favor de otra y esta quiere agradecerle haciéndole, a su vez, otro favor. En la Tr1 se conserva como calco la expresión "muchos otros clientes de mi abuelo sabían reenviar el ascensor (p. 123), como príncipes, justo cuando era necesario"; pero en español no es frecuente y resulta un poco forzada. En la SAT, la autora la transforma en "muchos otros clientes de mi abuelo sabían devolver un favor como príncipes, justo cuando era necesario" (p. 125), lo que explicita la metáfora y aporta claridad a la idea expresada.

\subsection{Introducción de elementos nuevos no presentes en el original}

En esta segunda categoría de transformaciones de semiautotraducción incluimos los siguientes fenómenos: explicitación, pérdida del cambio de lenguas, omisiones y adiciones.

1. Explicitación: la $A A A$ del original (p. 17) y de la primera traducción (p. 15) se convierte en "la Triple A" (p. 15) en la SAT. Si bien a continuación se explica que dicha sigla corresponde a "la Alianza Anticomunista Argentina", el cambio realizado permite leerlo como se expresa oralmente, aportando información más exacta para quien pudiese desconocer esta organización.

2. Pérdida del cambio de lenguas en el то: "C'est peut-être à cause de cette expression, vender gato por liebre" en el то (p. 84), aparece, en la Tr1, con una recreación del traductor como: "¿Será por ese dicho de 'vender liebre por gato'? ¿O era 'gato por liebre'? En fin". (p. 76), cambios que en la SAT se eliminan, volviéndose más apegado al original con: "¿Será por ese dicho de 'vender gato por liebre'?" (p. 78), pero con la evidente pérdida de la alternancia de lenguas.

3. Omisiones con respecto al to en la SAT: aquí hallamos dos tipos de eliminaciones, las de las referencias a la lengua española y las de expresiones con carga ideológica.

En cuanto a las primeras, encontramos un caso donde se elimina información. Podría interpretarse que la edición revisada por Alcoba de 2018, por un lado, tiene en cuenta a un lector que conoce el español hablado en Argentina; y, por otro, no sería necesario marcar la lengua, porque no hay alternancia de códigos. La frase en el TO, "ce terme espagnol", en alusión a la palabra "embute" (p. 51), vertido en Tr1 como "este término del idioma español, del habla argentina" (p. 47), es recortado en SAT como "este término" (p. 49). En Tr1, el traductor agrega una explicación sobre la localización del "término", teniendo en cuenta un lectorado amplio; en cambio, en SAT, la autora prefiere eliminar esas referencias, asumiendo que el lector conoce el contexto o que puede inferirlo.

Más adelante observamos, en el To, la expresión "aux doutes de tous les hispanophones" (p. 52), traducido literalmente en la Tr1 como "a las dudas de todos los hispanos parlantes" (p. 48), que se reduce con cambios en la sintaxis en SAT como "a todas las dudas" (p. 50).

En relación con el segundo tipo de eliminaciones, las omisiones de expresiones con carga ideológica, en el To encontramos: "mon père, qui était libre depuis longtemps déjà -il a été libéré quelques mois avant la guerre des Malouines, comme beaucoup de prisonniers politiques relâchés au moment où la dictature commençait à s'effondrer-". Allí, el término "prisioneros políticos liberados" (p. 134), se encuentra en la Tr1 como se resalta: "mi padre, en libertad desde poco después de la Guerra de Malvinas, cuando la dictadura había comenzado a derrumbarse y ya no pudo retener a los presos políticos" (p. 124). En este caso, hay un sentido que se agrega, el de no ser posible seguir encerrando 
personas por razones políticas. En cambio, en la SAT se elimina este concepto de los presos políticos liberados, que estaba presente en el original: "mi padre, en libertad desde hacía ya cierto tiempo, fue liberado unos meses antes de la Guerra de Malvinas, cuando la dictadura comenzaba a fisurarse". Esta alusión se refiere a los presos políticos que al igual que su padre fueron dejados en libertad un tiempo antes del advenimiento de la democracia, meses antes de la Guerra de Malvinas, que se desatara el 2 de abril de 1982.

4. Adiciones en la SAT de fragmentos que no existían en el To: en el to dice: "C'était étrange d'entendre ainsi, de nouveau, l'Ingénieur, derrière les mots de Dupin. Mais subitement, le fameux passage sur l'" excessive évidence " m'a glacée [...] Je le reproduis ici dans la traduction qu'en fit Charles Baudelaire" (p. 143). En Tr1 aparece: "Era extraño escuchar así, nuevamente, al Ingeniero, por detrás de las palabras de Dupin, en traducción de Charles Baudelaire. Pero súbitamente, al leer el famoso pasaje sobre la 'evidencia excesiva' quedé helada" (p. 132). Al respecto, en la SAT se pueden observar unos agregados explicativos:

Era extraño volver a escuchar al Ingeniero por detrás de las palabras de Dupin, que yo estaba leyendo en la traducción francesa de Charles Baudelaire, ya que era la que tenía a mano. Pero, de repente, al leer el famoso pasaje sobre la "excesiva evidencia”, quedé helada (p. 134).

En la SAT, por un lado, se aclara que se trata de una traducción francesa, lo que señala la diferencia de lenguas de los textos referidos en el relato; por otro, la autora agrega dos informaciones nuevas: que la narradora estaba leyendo ese cuento y que tenía a mano la versión en francés realizada por Baudelaire.

Otro ejemplo de adición es el siguiente. En el To, donde dice: "Elle a quitté le quartier peu de temps après" (p. 139), se conserva en la Tr1 como: "Poco tiempo después se fue del barrio" (p. 128). Pero en la SAT se agrega: "Poco tiempo después del ataque se fue del barrio" (p. 130), es decir, la adición de la palabra "ataque" refuerza el pasado traumático sufrido por los vecinos de la casa a partir de aquel brutal acontecimiento, el bombardeo de la casa de los conejos, que masacró a todos sus ocupantes.

\subsection{Introducción de elementos léxicos del español de Argentina}

Esta tercera categoría de transformaciones de semiautotraducción incluye la modificación, con respecto a la primera traducción, de elementos léxicos por otros más usados en Argentina, mediante el empleo de tiempos verbales, el uso del "vos", la omisión del sujeto y el uso de determinados términos empleados con mayor frecuencia allí.

1. Tiempos verbales: se ajustaron, en la SAT, dos tiempos verbales, el pasado compuesto y el futuro próximo del francés, a tiempos en español que corresponden a un uso más habitual en Argentina. Por un lado, el passé composé o pasado compuesto del original, traducido como pretérito perfecto compuesto en $\operatorname{Tr} 1$, se transforma en pretérito perfecto simple en la SAT. En el To dice: "je me suis enfin décidée" (p. 14); en Tr1, "me he decidido" (p. 12), y en la SAT, "me decidí" (p. 14). En nuestro contexto argentino, se usa con mayor frecuencia este último tiempo verbal, es más usado que el de las formas compuestas. En este sentido, entonces, la SAT vuelve el texto más natural para el receptor argentino, orientado a la variedad de español de esta zona de América del Sur.

Por otra parte, el futur proche en francés en el то: "nous allons vivre dans la clandestinité" (p. 17), aparece en futuro simple en 
Tr1: "viviremos en la clandestinidad", y se cambia por la misma construcción del TO en la SAT: "ir a + infinitivo" en "vamos a vivir en la clandestinidad" (p. 17).

2. "Vos": introducción del uso del pronombre "vos" en la SAT, no utilizado en la primera traducción. En el TO, "comme tu voulais" (p. 15), en Tr1 "como querías", se vuelve en la SAT: "como vos querías" (p. 14). Como lo indica el Diccionario Panhispánico de Dudas de la Real Academia Española, lo que se conoce como "voseo" es "el uso de formas pronominales o verbales de segunda persona del plural (o derivadas de estas) para dirigirse a un solo interlocutor. Es propio de distintas variedades regionales del español americano e implica acercamiento y familiaridad".

3. Omisión del pronombre sujeto: el español favorece la omisión de pronombres sujeto, a diferencia del francés, donde deben ser siempre expresados. Donde en el to dice: "J'écoute en silence" (p. 17), en $\operatorname{Tr} 1$ se conserva el sujeto "Yo escucho en silencio" (p. 15), y en la SAT se suprime: "La escucho en silencio" (p. 17), resultando la construcción más natural a los oídos de los hispanohablantes.

4. Variedades de español de Argentina: dentro de esta subcategoría observamos diversos tipos de cambios. En primer lugar, el empleo de términos más afectivos, como en "un petit garçon" (p. 20) del TO, "niño" (p. 17) en la Tr1, que cambia a "nene" (p. 19), un término más afectivo y coloquial utilizado en este país. Podíamos decir que en el habla cotidiana rioplatense, en la que se inscriben los usos de Laura Alcoba oriunda de la ciudad de La Plata, se usa con más frecuencia "nene" o "nena", y se prefiere "niño" para usos más formales, como advertencias, como, por ejemplo, en "solo apto para niños".
En otro caso, "Il veut" (p. 121), pronunciado por la madre de Laura, en Tr1 se explicita el pronombre con "papá quiere" (p. 119) y en la SAT se transforma en "Mi viejo quiere" (p. 121), un término muy usado en nuestro país, familiar y cariñoso (Zorrilla, 2018), con el agregado del posesivo que acentúa aún más ese carácter afectuoso.

En segundo lugar, se observa el empleo de términos de registro familiar o coloquial, como con el verbo "discuter" (p. 38) del Tо, "discutir" (p 36) en Tr1, donde se cambia ligeramente el sentido, porque su significado es "conversar, hablar" en español, y que es transformado en SAT por "charlar" (p. 38), un término que se acerca más a la idea del original $\mathrm{y}$, además, le agrega un matiz de registro coloquial muy usado en Argentina.

En último lugar, encontramos la introducción de términos regionales, más usados en Argentina: "conduire" (p. 24), "conducir" (p. 21) en Tr1, se precisa como "manejar" (p. 23), utilizado para designar la acción de conducir un automóvil.

\section{Conclusiones}

Abordamos el apasionante caso de una escritora argentina que por haberse instalado en Francia desde pequeña recibe toda su educación en lengua francesa y, como resultado, su lengua de escritura es el francés. Pero lo que hace muy especial este caso de semiautotraducción es que no solo el español es la lengua primera de la autora, sino que el relato, una autobiografía ficcional, se basa en la experiencia vivida en su lengua materna.

Nos interesa destacar el interés que representa para un estudio traductológico comparar el original, Manèges, que cuenta una petite histoire argentine en francés, para un público francófono que no necesariamente conoce aquella 
época de dictadura militar y la clandestinidad que padeció el personaje de la niña Laura, con su primera traducción al español, La casa de los conejos, y los cambios que "necesita" hacer la autora, en vistas de lograr ese tono, esa voz, esos detalles, y así poder leer su obra de una manera más satisfactoria.

Para comprender en profundidad el objeto de estudio de este trabajo, los cambios realizados por la autora a la primera traducción al español de su novela en francés Manèges, fue preciso sumergirnos, en una primera etapa, en los desarrollos teóricos sobre las nociones que se acercaban al fenómeno analizado, el de las traducciones donde participan en mayor o menor medida los propios autores. Por un lado, la autotraducción o traducción donde interviene el autor. Pero al tratarse de una revisión de una primera traducción, también fue necesario, por otro lado, retomar la noción de retraducción a cargo de los autores. Aunque pudimos identificar una tendencia a regresar al texto fuente para restituir sus especificidades con precisión, también se observaron cambios que introdujeron elementos nuevos, así como se eliminaron otros existentes en el original.

Otro aspecto para destacar de esta traducción revisada por la autora es la intertextualidad. Como señalamos al comienzo, la autoría de esta traducción se presenta con matices. Por un lado, en un primer plano, el nombre del traductor que figura en la portada es el de Leopoldo Brizuela. Por otro, en un plano menos visible, la intervención de la autora como revisora de la traducción aparece en la contratapa. Esta información se desprende, además, del prólogo de un reconocido escritor francés, Daniel Pennac, que introduce esta nueva edición y da indicios de la intervención de la autora en el nuevo texto. $\mathrm{Y}$, por último, de los relatos de la propia Alcoba en conferencias y conversatorios, donde explica su proceso de revisión de la traducción, como los que hemos comentado anteriormente.

Asimismo, recorrimos las diversas funciones que puede cumplir la retraducción debido a su carácter polimorfo, como lo caracterizó Liliane Rodríguez (1990), en comparación con las atribuidas al dios Mercurio. Hemos observado que, en este caso, las funciones predominantes son la de "vendedor", porque se procura preservar los detalles del original y la publicación de la retraducción constituye un argumento de venta; y la de "mensajero", que siente la necesidad de adaptar el mensaje a otro momento y a otros lectores.

Con respecto a nuestra primera hipótesis, verificamos la necesidad de ajustar las categorías de "autotraducción" y "retraducción" a los casos de estudio y, al final del recorrido teórico, presentamos la noción que mejor se ajustaba a nuestro análisis, la de semiautotraducción, de Xosé Manuel Dasilva (2016), dentro de la subcategoría de "traducción alógrafa", revisada por el autor. En el proceso de traducción, Alcoba revisó el escrito, la traducción efectuada por el traductor, en solitario. Una vez finalizada la revisión, Brizuela autorizó los cambios. Es importante recordar que la voluntad de intervenir y revisar el texto no fue impuesta por la editorial, sino que tuvo su origen en la necesidad de la propia autora de hacer algunos cambios.

Luego de estos apartados teóricos, pasamos, en una segunda etapa, a la metodología y al análisis del corpus seleccionado. Nos basamos en la propuesta de Patricia López López-Gay (2008) de transformaciones de autotraducción, para analizar los cambios introducidos en la segunda traducción revisada con respecto a la primera y en relación con el original. Una vez detectados y registrados los cambios (de manera manual, en una tabla o planilla de cálculo), en la nueva versión revisada o "semiautotraducción" (SAT), como la hemos llamado en este trabajo, los agrupamos en tres tendencias.

La primera, y una de las más destacadas, es la de aspirar a una mayor precisión con respecto al original que en la primera traducción. Esto se refleja, en primer lugar, por un mayor acercamiento al original, que puede observarse mediante diversas estrategias: 1) eliminación 
de adiciones de la primera traducción que no existían en el texto fuente; 2) cambio de términos con carga ideológica más pesada en la primera traducción, por otros más neutros y cercanos al original; 3) eliminación de términos vulgares agregados en la primera traducción; y 4) restitución de términos ideológicamente significativos que fueron neutralizados en la primera traducción.

En segundo lugar, el mayor grado de detalle se observa en la reposición de elementos, como fragmentos de más de cuatro líneas, que fueron totalmente borrados en la primera versión. Podemos interpretar que esta reposición de elementos está directamente relacionada con la participación de la autora y su acercamiento al original, y con el tiempo transcurrido, diez años entre ambas ediciones, lo que hace que el público objetivo de cada publicación sea distinto. En efecto, para el nuevo lectorado, será de gran interés acceder a los detalles ideológicamente relacionados con la última dictadura militar que se neutralizaron en la edición del 2008.

En tercer lugar, la precisión se lleva a cabo mediante mecanismos de ajuste de sentido: 1) sentido diferente; 2) sentido restringido; 3) falso sentido y 4) sentidos metafóricos.

La segunda tendencia consistió en introducir, en la SAT, elementos nuevos no presentes en el texto fuente, por medio de los siguientes mecanismos: 1) explicitación: cambios que tienden a ofrecer una información más clara para un lector que desconoce el contexto histórico; 2) pérdida del cambio de lenguas: se trata de un fenómeno recurrente a lo largo del texto, puesto que en el original hay más de una veintena de referencias culturales que se expresan en español y en la traducción no se percibe esa alternancia de código; 3) omisiones, en la SAT, de elementos del original: como referencias a la lengua española, que podrían resultar innecesarias ante un lector hispanoparlante, o expresiones con carga ideológica; y 4) adiciones, en la SAT, de elementos inexistentes en el original: agregados explicativos donde se ofrece mayor detalle, producto probablemente de la evocación de los recuerdos de la autora cuando relee su historia.

La tercera tendencia que detectamos en esta traducción revisada es la de plasmar y reforzar la identidad argentina. Y esto nos remite a las ideas de Venuti (2013) y de Spoturno (2018) sobre la identidad diferenciada que permite proyectar una retraducción, es decir, en nuestro caso estudiado, nos permite comprender esa fuerza que moviliza a Laura a reimprimir, en la nueva edición, una imagen más argentinizada, con la puesta en valor de elementos lingüístico-culturales propios de la cultura argentina y que marcan una diferencia con respecto a la primera traducción.

Esta inclinación se manifiesta mediante diversos procesos, que acentúan el uso de la variedad de español rioplatense hablado en Argentina: 1) cambios a tiempos verbales preferentemente usados en esta región (por ejemplo, el pretérito perfecto simple en lugar del pretérito perfecto compuesto, lo que muestra la predilección por la forma simple del pasado en lugar de la compuesta); 2) introducción del pronombre "vos" en la SAT, que evoca el voseo, que consiste en reemplazar el pronombre "tú", de segunda persona del singular, con el pronombre "vos", como fórmula de tratamiento familiar y afectivo dirigida a una sola persona (Zorrilla, 2018); 3) omisión del pronombre sujeto, sujeto elíptico, omitido o nulo (Devís Márquez, 2011), cuando en la oración no existe ningún sujeto explícito, pero se puede identificar cuál es a través de la conjugación del verbo - el español es una lengua pro-drop, del inglés pronoun-dropping, que omite el pronombre, al contrario del francés, que exige la presencia de un sujeto (Bosque y Gutiérrez-Rexach, 2009)—; 4) cambios por variedades de español más usadas en Argentina: términos coloquiales, afectivos y regionales.

De esta manera, es momento de destacar que pudimos comprobar nuestra segunda hipótesis, la de afirmar que, en este tipo de traducciones 
con participación del autor, la libertad que le brinda su privilegiada posición se desplaza entre dos polos, el alejamiento y el acercamiento con respecto al texto fuente, entre la introducción de elementos nuevos que no se leen en el texto original y la búsqueda de mayor precisión que en la primera traducción.

Dentro de este movimiento de recreación de la autora en su reescritura como traductora, observamos la tendencia a agregar elementos con respecto a la primera traducción, que impregnan el texto de la variedad de español rioplatense en Argentina.

Además, es preciso señalar también que la libertad que se asocia a los autores que traducen sus propias obras, igualmente pudo observarse en manos del traductor, Leopoldo Brizuela, en los cambios que introdujo en la primera traducción que no se leen en el texto original.

Las tendencias que sugieren las transformaciones observadas, desde las más sutiles de cambio de registro hasta las más notorias, como las adiciones, omisiones o restituciones de fragmentos, son percibidas por quien compara los tres textos, el original y las dos traducciones. Esto implica que se trata de un terreno a explorar rico para la traductología, donde las investigaciones sobre los casos de estudio echan luz sobre los diversos factores y propósitos que dan origen a las retraducciones o revisiones de traducción donde intervienen los autores, así como las variadas estrategias que se ponen en marcha.

También significa que la visibilidad de estas nuevas versiones está disponible solo para los traductólogos, los traductores, los profesores de traducción, expertos en ediciones críticas; pero ¿qué sucede con el lector meta? Sería interesante comparar la recepción de ciertos elementos por parte de diferentes públicos. No podemos responder a este interrogante, pues sería necesario otro tipo de estudio sobre la recepción de la autotraducción. Pero sí podríamos afirmar que la recepción de quien leyera la segunda traducción o semiautotraducción de Laura Alcoba, en comparación con que quien solo hubiese leído la primera traducción, accederá a detalles identitarios de Argentina más marcados que aquel que haya leído sólo la primera traducción.

Como ninguna traducción es inocente, sino que persigue una intención, las retraducciones llevadas a cabo por el mismo autor, las autotraducciones y las semiautotraducciones representan una valiosa oportunidad para que autores, y autotraductores, en tanto sujetos históricos, actualicen sus propias obras.

\section{Referencias}

Alcoba, L. (2007). Manèges. Petite histoire argentine. Gallimard.

Alcoba, L. (2008). La casa de los conejos (Leopoldo Brizuela, Trad.). Edhasa.

Alcoba, L. (2013). Le bleu des abeilles. Gallimard.

Alcoba, L. (2014). El azul de las abejas (Leopoldo Brizuela, Trad.). Edhasa.

Alcoba, L. (2017). La danse de l'araignée. Gallimard.

Alcoba, L. (2018a). La casa de los conejos (Daniel Pennacm Prólogo; Leopoldo Brizuela, Trad.). Edhasa.

Alcoba, L. (2018b). La danza de la araña (Mirta Rosenberg y Gastón Navarro, Trad.) Edhasa.

Berman, A. (1990). La retraduction comme espace de la traduction. Palimpsestes, 4, 1-7. https:// doi.org/10.4000/palimpsestes.596

Bosque, I. y Gutiérrez-Rexach, J. (2009). Fundamentos de sintaxis formal. Ediciones Akal.

Dancette, J. (1989). La faute de sens en traduction. TTR : Traduction, Terminologie, Rédaction, 2(2), 83-102. https://www.erudit.org/fr/revues/ ttr/1989-v2-n2-ttr1471/037048ar/

Dasilva, X. M. (2016). En torno al concepto de semiautotraducción. Quaderns : revista de traducció, (23), 15-35. https://raco.cat/index.php/ QuadernsTraduccio/article/view/310614

Delisle, J. (1993). La traduction raisonnée. Manuel d'initiation à la traduction professionnelle de 
l'anglais vers le français. Presse de 1'Université d'Ottawa.

Devís Márquez, P. P. (2011). El parámetro del sujeto nulo y la enseñanza del español como lengua extranjera. Reflexión gramatical. Didáctica, Lengua y Lieratura, 23, 59-86. http://dx.doi. org/10.5209/rev_DIDA.2011.v23.36310

Gambier, Y. (1994). La retraduction, retour et détour. Meta, 39(3), 413-417. https://doi. org/10.7202/002799ar

Grutman, R. (1998). Autotranslation. En M. Baker (Ed.), Routledge encyclopedia of translation studies (pp. 17-20). Routledge.

Grutman, R. (2007). L'écrivain bilingue et ses publics : une perspective comparatiste. En A. Gasquet y M. Suárez (Eds.), Ecrivains multilingues et écritures métisses : L'hospitalité des langues (pp. 3150). Presses Universitaires Blaise-Pascal.

Grutman, R. (2009). La autotraducción en la galaxia de las lenguas. Quaderns. Revista de Traducció, (16), 123-134. https://www.researchgate.net/ publication/44813709_La_autotraduccion_ en_la_galaxia_de_las_lenguas

Grutman, R. (2013). Autotraduction, asymétrie extraterritorialité. In C. Lagarde et $\mathrm{H}$. Tanqueiro (Ed.), L'autotraduction aux frontières de la langue et de la culture (pp. 37-44). Editions Lambert-Lucas.

Hurtado Albir, A. (2013 [2001]. Traducción y traductología. Introducción a la traductología. Cátedra.

López López-Gay, P. (2008). La autotraducción literaria: traducibilidad, fidelidad, visibilidad. Análisis de las autotraducciones de Agustín Gómez-Arcos y Jorge Semprún. Université Paris Diderot, Universidad Autónoma de Barcelona.

López López-Gay, P. (n. d.). La autotraducción como fuente de reflexión para la traducción profesional. I Congreso Internacional de Traducción Especializada. http://www.bibliotecact.com.ar/ PDF/02254.pdf

Manterola Agirrezabalaga, E. (2014). La literatura vasca traducida. Peter Lang.

Molina, L. (2006). El otoño del pingüino: análisis descriptivo de la traducción de los culturemas. Publicaciones de la Universitat Jaume I.
Palazuelos, J. C., Vivanco, H., Hörmann, P. y Garbarini, C. G. (1992). El error en traducción. Pontificia Universidad Católica de Chile. https://id.erudit.org/iderudit/001992ar

Parcerisas I Vazquez, F. (2007). Idéologie et autotraduction entre cultures asymétriques. Atelier de Traduction, (7), 111-119. https:// usv.ro/fisiere_utilizator/file/atelierdetraduction/arhive/AT/AT\%20NUMEROS/ AT\%207/7_111-119_Francesc\%20Parcerisas $\% 20 I \% 20$ Vasquez $\% 20$ (Espagne) $\% 20-\% 20$ Id\%C3\%A9ologie\%20et\%20autotraduction $\% 20$ entre $\% 20$ cultures $\% 20$ asym $\% \mathrm{C} 3 \% \mathrm{~A}$ 9triques.pdf

Popovic, A. (1976). Dictionary for the analysis of literary translation. University of Alberta.

Popovic, A. (2011). The concept "shift of expression" in translation analysis. In J. S. Holmes (Ed.), The nature of translation. Essays on the theory and practice of literary translation (pp. 78-87). De Gruyter Mouton.

Recuenco Peñalver, M. (2013). Traducción y autotraducción. El caso de Vasilis Alexakis [Tesis de doctorado]. Universidad de Málaga.

Rodriguez, L. (1990). Sous le signe de Mercure, la retraduction. Palimpsestes, 4, 63-80. https:// doi.org/10.4000/palimpsestes. 604

Santoyo, J. C. (2002). Traducciones de autor: una mirada retrospectiva. Quimera, (210), 27-32.

Santoyo, J. C. (2005). Autotraducciones: una perspectiva histórica. Meta, 50(3), 858-867. https://doi.org/10.7202/011601ar

Schleiermacher, F. (2000). Sobre los diferentes métodos de traducir. Traducción y comentarios de Valentín García Yebra. Edición bilingüe. Gredos.

Spoturno, M. L. (2010). Efectos y dinámicas de lectura en la traducción de voces minoritarias. El caso de Sandra Cisneros, Shulamis Yelin y Stevie Smith. IV Congreso Internacional de Letras, 1246-1253. http://2010.cil.filo.uba.ar/ponencia/efectos$\mathrm{y}$-din $\% \mathrm{C} 3 \% \mathrm{~A} 1 \mathrm{micas}$-de-lectura-en-la-traducci $\%$ C3\%B3n-de-voces-minoritarias-el-caso-desandra-cis

Spoturno, M. L. (2018). Self-retranslation as a rite of passage: Rosario Ferré's English version of "La muñeca menor. Mutatis Mutandis, 11(2), 
356-375. https://doi.org/10.17533/udea. mut.v11n2a04

Steiner, G. (2009). Extraterritorial. Ensayos sobre la literatura y la revolución del lenguaje. Adriana Hidalgo editora.

Tanqueiro, H. (1999). Un traductor privilegiado: el autotraductor. Quaderns. Revista de traducció, (3), 19-27. http://www.raco.cat/ index.php/QuadernsTraduccio/article/ download/25182/25016

Tanqueiro, H. (2007). L'autotraduction comme objet d'étude. Atelier de Traduction, 7, 101109. https://usv.ro/fisiere_utilizator/file/ atelierdetraduction/arhive/AT/AT\%20 NUMEROS / AT\%207/7_101-109_He1ena $\% 20$ Tanqueiro $\% 20$ (Espagne) $\% 20$
- $\% 20 \mathrm{~L} \% \mathrm{E} 2 \% 80 \% 99$ autotraduction $\% 20$ comme $\% 20$ objet $\% 20 \mathrm{~d} \% \mathrm{E} 2 \% 80 \% 99 \%$ C3\%A9tude.pdf

Tanqueiro, H. (2011). Sobre la autotraducción de referentes culturales en el texto original: la autotraducción explícita y la autotraducción in mente. En X. M. Dasilva y H. Tanqueiro (Eds.), Aproximaciones a la autotraducción (pp. 245-259). Editorial Academia del Hispanismo.

Venuti, L. (2013). Retranslations: The creation of value. In Translation changes everything. Theory and practice (pp. 96-108). Routledge.

Zorrilla, A. M. (2018). Diccionario normativo del español de la Argentina. Editorial Colegio de Traductores Públicos de la Ciudad de Buenos Aires.

Cómo citar este artículo: Ghirimoldi, M. E. (2022). Traducción revisada por la autora: la semiautotraducción en La casa de los conejos de Laura Alcoba. Mutatis Mutandis, Revista Latinoamericana de Traducción, 15(1), 111-129. https://doi.org/10.17533/udea.mut.v15n1a07 